\title{
Sequential Treatment of Metastatic Adenocarcinoma of the Pancreatic Duct with Liver Metastasis Following the NAPOLI-1 Study Protocol with nal-Irinotecan plus 5-FU in the Second Line
}

\author{
Dilara Akhoundova Sanoyan ${ }^{a} \quad$ Cäcilia S. Reiner ${ }^{\text {b }}$ \\ Panagiota Papageorgiou ${ }^{c} \quad$ Alexander R. Siebenhüner $^{a}$ \\ aDepartment of Medical Oncology and Hematology, University Hospital Zurich and \\ University of Zurich, Zurich, Switzerland; 'bnstitute for Diagnostic and Interventional \\ Radiology, University Hospital Zurich and University of Zurich, Zurich, Switzerland; \\ ${ }^{c}$ Comprehensive Cancer Center Zurich, University Hospital Zurich, Zurich, Switzerland
}

\section{Keywords}

Metastasis - Adenocarcinoma - Pancreatic cancer - FOLFIRINOX - nal-irinotecan · nab-paclitaxel $\cdot$ Gemcitabine $\cdot$ Sequential treatment

\begin{abstract}
Pancreatic ductal adenocarcinoma (PDAC) is typically diagnosed at an advanced or metastatic stage, when curative surgery is not recommended. Therefore, the prognosis is poor for this dismal disease, with only $1-2 \%$ of the patients reaching the 5 -year survival follow-up. Current advances in systemic treatment with gemcitabine regimens, specifically polychemotherapy with gemcitabine plus nab-paclitaxel or other multidrug regimens such as FOLFIRINOX in the first line, have improved disease control over time. This higher efficacy of systemic treatment enables metastatic PDAC patients to receive second-line treatment more often nowadays. Currently, there is only one regimen for second-line treatment approved by the EMA, FDA, and Swissmedic, based on the phase III NAPOLI-1 study. In this case report, we present an outstanding response to sequential treatment with gemcitabine plus nab-paclitaxel followed by second-line treatment with nal-irinotecan plus 5-fluorouracil. ( 2020 The Author(s)




\section{Introduction}

Pancreatic ductal adenocarcinoma (PDAC) is typically diagnosed at an advanced or metastatic stage, when curative surgery is not recommended. Therefore, the prognosis is poor for this dismal disease, with only $1-2 \%$ of the patients reaching the 5-year survival follow-up [1, 2]. Current advances in systemic treatment with gemcitabine regimens, specifically polychemotherapy with gemcitabine plus nab-paclitaxel or other multidrug regimens such as FOLFIRINOX in the first line, have improved disease control over time [3]. This higher efficacy of systemic treatment enables metastatic PDAC (mPDAC) patients to receive second-line treatment more often nowadays. Currently, there is only one regimen for second-line treatment approved by the EMA, FDA, and Swissmedic, based on the phase III NAPOLI-1 study [4]. In this case report, we present an outstanding response to sequential treatment with gemcitabine plus nab-paclitaxel followed by second-line treatment with nal-irinotecan plus 5-fluorouracil (5-FU).

\section{Case}

A 48-year-old Caucasian male patient presented to a regional hospital with a 3-week history of obstructive jaundice, pruritus, tea-colored urine, acholic stools, and fatigue. His weight was reported to be stable at that time, with obesity grade 1 and a BMI of $32.8 \mathrm{~kg} / \mathrm{m}^{2}$. Collectively, his medical history included insulin-dependent diabetes mellitus, arterial hypertension, and moderate obstructive sleep apnea syndrome as complications of his smoking history of 20 cumulative pack-years. He denied regularly drinking alcohol. He had no history of cancer and no tumors were noticed in his family.

Ultrasonography revealed dilated extrahepatic bile ducts with a hypoechogenic tumor at the pancreatic head. A following computed tomography (CT) scan revealed multiple enlarged peripancreatic lymph nodes and a tumor mass $(23 \times 15 \mathrm{~mm})$ at the pancreatic head with no other tumor manifestations at that time. The tumor marker carbohydrate antigen 19-9 (CA19-9) was elevated at 5,700 kU/L (normal <24) and bilirubin was at $104 \mu \mathrm{mol} / \mathrm{L}$ (normal $<21$ ). Autoimmune pancreatitis was ruled out by a normal serum immunoglobulin G4 level.

The patient was transferred to our center for further diagnostics and interdisciplinary discussion of treatment. A following magnetic resonance imaging (MRI) scan of the liver and a fluorodeoxyglucose positron emission tomography-CT scan revealed a metabolically active tumor at the pancreatic head and multiple liver metastases (Fig. 1). Endoscopic retrograde cholangiopancreatography with stenting was performed, and fine-needle aspiration biopsy at the time of the endoscopic ultrasound demonstrated a cytology consistent with adenocarcinoma. During discussion within the multidisciplinary tumor board, all members recommended the start of systemic chemotherapy.

At the time of diagnosis, the patient's performance status (PS) was evaluated according to the Eastern Cooperative Oncology Group (ECOG) and was reported as grade 1: restricted in physically strenuous activity, but ambulatory and able to carry out light work. The CA19-9 levels decreased adequately to $295 \mathrm{kU} / \mathrm{L}$ after stenting of the extrahepatic bile ducts as compared to the baseline value of the tumor marker before initiating systemic treatment. First-line treatment with the combination of nab-paclitaxel plus gemcitabine based on the MPACT [5] protocol was applied as the patient's ECOG PS (at 1) and the bilirubin levels (at 54 $\mu \mathrm{mol} / \mathrm{L})$ were still elevated.

The patient tolerated 6 cycles of this chemotherapy combination, and restaging documented stable disease of the primary tumor and liver metastases on CT scans of the chest, abdomen, and pelvis and on MRI, respectively. The patient received a further 3 cycles of nab- 
Akhoundova Sanoyan et al.: Sequential Treatment for Metastatic Pancreatic Cancer

A

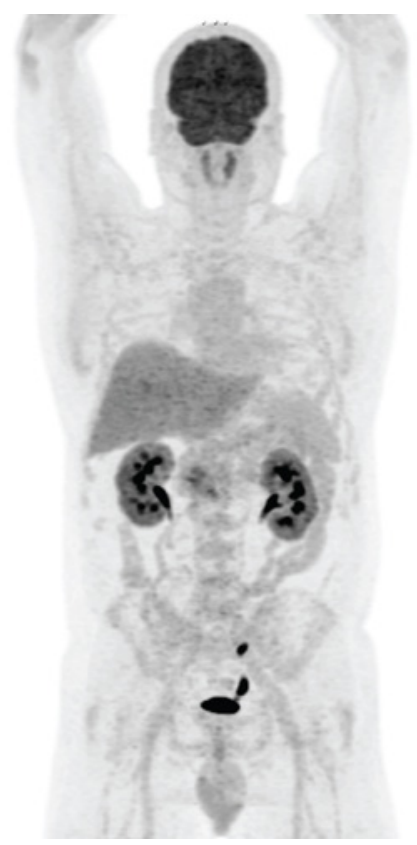

B



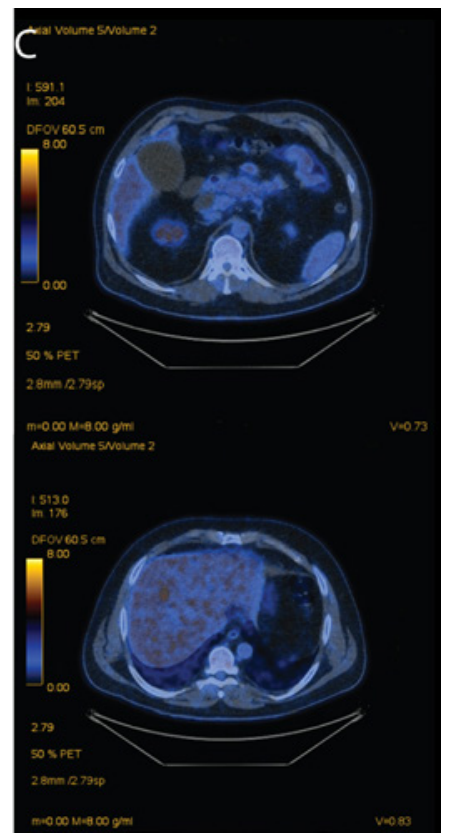

Fig. 1. Baseline partial-body positron emission tomography/computed tomography scan with 301-MBq fluorodeoxyglucose $\left({ }^{18} \mathrm{~F}\right.$-FDG). A, B Coronal (A) and sagittal plane (B) showing moderately increased FDG accumulation in the pancreatic head corresponding to pancreatic carcinoma. $\mathbf{C}$ Axial plane showing a 3-cm hypodense lesion in the pancreatic head with moderately increased FDG accumulation.

paclitaxel plus gemcitabine. Unfortunately, after these 9 cycles of first-line treatment, the CA19-9 levels rose to $680 \mathrm{kU} / \mathrm{L}$ without signs of bile duct obstruction, and the tumor at the pancreatic head and the liver metastases were radiologically progressive on CT of the chest, abdomen, and pelvis and on MRI of the liver, respectively (Fig. 2b). At this stage, the progression after first-line treatment was discussed with the patient, and second-line treatment with nal-irinotecan in combination with 5-FU analogs according to the NAPOLI-1 protocol was planned.

The ECOG PS remained stable at 1, supporting a continuation of this combination of nalirinotecan and 5-FU. Shortly after 2 cycles of this combination, the CA19-9 level dropped to $230 \mathrm{kU} / \mathrm{L}$, and planned restaging after 6 cycles revealed a partial response of the primary tumor and of the liver metastases on CT of the chest, abdomen, and pelvis and on liver MRI, respectively (Fig. 2c, d). This treatment success could be documented for all the following cycles, and recently, this patient has received the seventeenth cycle of second-line nal-irinotecan plus 5-FU. At the latest radiological and clinical evaluation, the CA19-9 level had dropped to $72 \mathrm{kU} / \mathrm{L}$, and stable disease was still noticed in comparison to the response after 6 cycles. The patient did not suffer from any severe grade 3 or 4 toxicities, and it is worth noticing that the grade 2 anemia previously observed during first-line treatment had completely reverted to normal hematologic blood values.

To the best of our knowledge, this is one of the most extraordinary responses of mPDAC to treatment according to MPACT and NAPOLI-1 protocols; it even extends their presented progression-free survival (PFS) and overall survival (OS) rates by more than 4 months with a current PFS of 8.0 months and an OS of 17 months. Of note, we highlight that the quality of life (QoL) was high in this young and fit patient. 

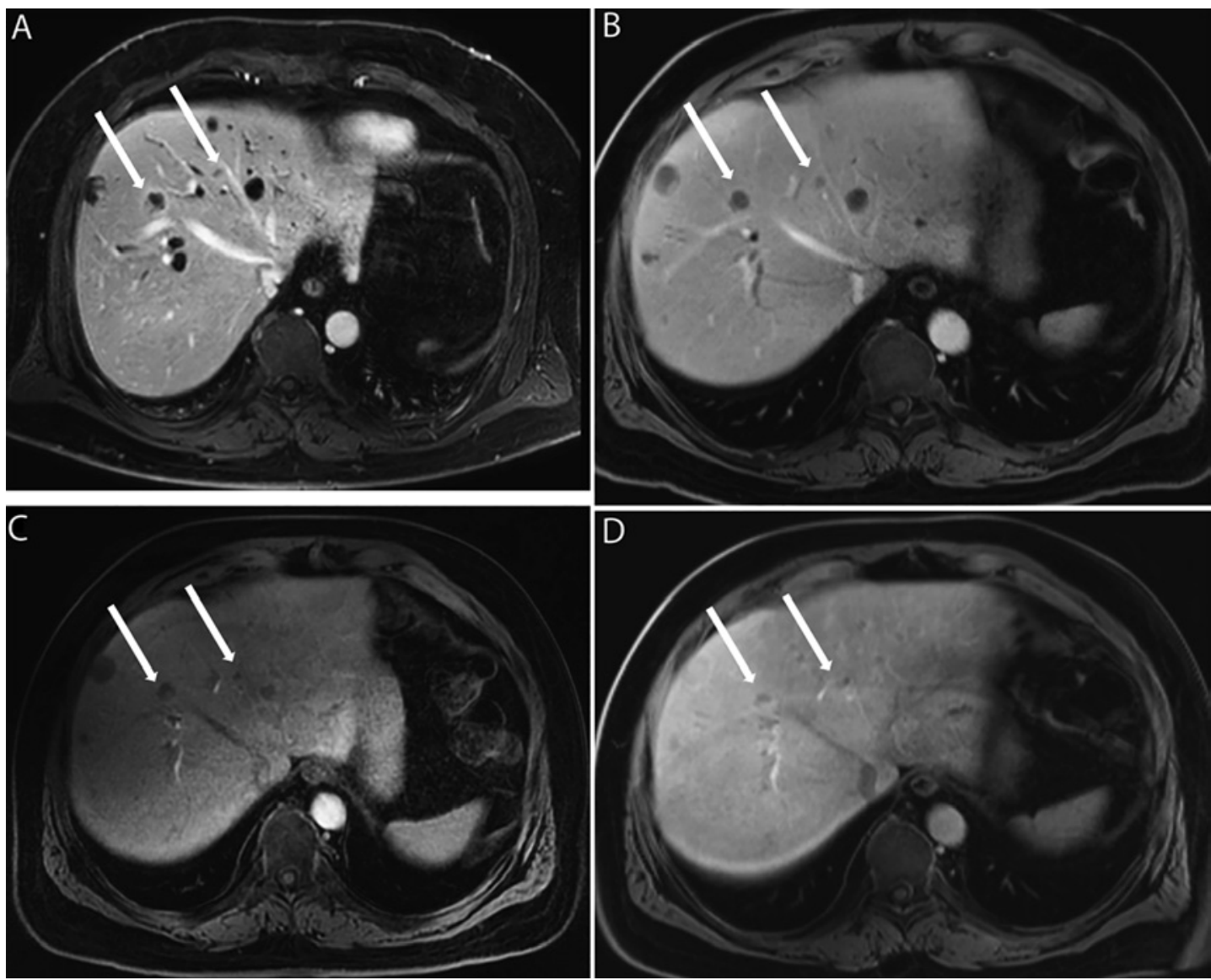

Fig. 2. Abdominal magnetic resonance images showing multiple lesions in liver segments II, IVa, IVa/VIII, and VIII with 2 representative liver metastases shown in segment VIII (arrows). A At diagnosis, there were 2 representative lesions 15 and $4 \mathrm{~mm}$ in size in segment VIII. B On progression after first-line chemotherapy with nab-paclitaxel/gemcitabine, the 2 lesions measured 14 and $8 \mathrm{~mm}$. C Three months after treatment with second-line chemotherapy with nal-irinotecan/5-fluorouracil (5-FU), the lesions in liver segment VIII had regressed to 13 and $7 \mathrm{~mm}$. D Eight months after treatment with second-line chemotherapy with nalirinotecan/5-FU, the lesions in liver segment VIII had regressed to 11 and $5 \mathrm{~mm}$.

\section{Discussion}

Algorithms for sequential management of patients with mPDAC based on expert opinions are emerging. The availability of more effective first-line treatments (the combination of nabpaclitaxel plus gemcitabine and FOLFIRINOX based on the results of phase III studies) allows the development of second-line treatment options based on the results of phase II and III studies for patients with mPDAC [4-8]. On the other hand, the PANCREOX study did not demonstrate any difference in PFS or OS between infusional 5-FU/leucovorin (LV) and mFOLFOX6 (infusional 5-FU/LV and oxaliplatin), questioning the role of the addition of oxaliplatin for the management of patients previously treated with gemcitabine-based regimens [9].

Collectively, the results of the NAPOLI-1 study, which analyzed the effect of nal (nanoliposomal)-irinotecan alone or combined with 5-FU/LV in a phase III trial on patients with mPDAC previously treated with gemcitabine-based therapies, showed that this regimen 
prolongs survival (6.1 vs. 4.2 months), with a manageable safety profile [4]. Similar to other diseases like colon cancer, for example, the availability of second- and even third-line treatments implies that the management of the disease should be viewed as a continuum of care with several lines of treatment rather than as compartmentalized treatments. However, there are no clear recommendations in the different guidelines available (e.g., NCCN or ESMO) regarding the optimal sequence of treatment for mPDAC. One of the main reasons is the lack of parallel comparison between possible approaches such as FOLFIRINOX and gemcitabine plus nab-paclitaxel or nal-irinotecan plus 5-FU in sequential treatments.

Due to the lack of randomized trials in the second-line setting after the combination of nab-paclitaxel plus gemcitabine as first-line treatment, one approach to determining results of second-line treatment is to analyze the outcomes of the patients who have received secondline treatment after a first line with the combination of nab-paclitaxel plus gemcitabine in the MPACT trial [10].

One of the main goals of treating metastatic pancreatic cancer is to maintain healthrelated QoL. Recently published data from the NAPOLI-1 study have demonstrated no deterioration from baseline on most of the health-related QoL subscales (e.g., fatigue or physical or cognitive function), while survival was significantly prolonged [11].

Currently, there are no good predictive molecular markers favoring one chemotherapy regimen over any of the other available options. Moreover, no promising molecular markers for the personalized treatment of pancreatic cancer are available. Therefore, factors to be considered in making treatment decisions include the ECOG PS, comorbidities, residual toxicities (i.e., neuropathy), prior treatments, and the patient's goals and preferences. In summary, this case report highlighted factors such as a heightened ECOG PS, elevated bilirubin at baseline, and diabetes in favor of the nab-paclitaxel-plus-gemcitabine regimen instead of FOLFIRINOX as first-line treatment.

\section{Conclusion}

Sequential treatment of metastatic pancreatic cancer will change survival rates with this dismal disease. Here, we could show a quite sufficient and effective sequence for a young and fit patient following MPACT and NAPOLI-1 study protocols with which the patient's QoL remained high.

\section{Acknowledgement}

We would like to thank the patient for giving us consent to publish the case report.

\section{Statement of Ethics}

Written informed consent was provided by the patient to publish this case (including publication of images).

\section{Disclosure Statement}

The authors have no conflicts of interest to disclose. 
Akhoundova Sanoyan et al.: Sequential Treatment for Metastatic Pancreatic Cancer

\section{Funding Sources}

No funding was received for this case report.

\section{Author Contributions}

D.A.S. wrote the manuscript and gave critical approval; C.S.R. designed the figures, read the manuscript, and gave critical approval; P.P. edited the manuscript and gave critical approval; A.R.S. designed the case report, wrote the manuscript, edited the figures, and gave critical approval.

\section{References}

1 American Cancer Society. Cancer facts \& figures 2019 [Internet]. Atlanta: American Cancer Society; 2019. Available from: https://www.cancer.org/research/cancer-facts-statistics/all-cancer-facts-figures/cancerfacts-figures-2019.html.

2 Tempero MA. NCCN guidelines updates: pancreatic cancer. J Natl Compr Canc Netw. 2019;17(5.5):603-5.

3 Ducreux M, Seufferlein T, Van Laethem JL, Laurent-Puig P, Smolenschi C, Malka D, et al. Systemic treatment of pancreatic cancer revisited. Semin Oncol. 2019;46(1):28-38.

4 Wang-Gillam A, Li CP, Bodoky G, Dean A, Shan YS, Jameson G, et al. Nanoliposomal irinotecan with fluorouracil and folinic acid in metastatic pancreatic cancer after previous gemcitabine-based therapy (NAPOLI-1): a global, randomised, open-label, phase 3 trial. Lancet. 2016;387(10018):545-57.

5 Von Hoff DD, Ervin T, Arena FP, Chiorean EG, Infante J, Moore M, et al. Increased survival in pancreatic cancer with nab-paclitaxel plus gemcitabine. N Engl J Med. 2013;369(18):1691-703.

6 Conroy T, Desseigne F, Ychou M, Bouché O, Guimbaud R, Bécouarn Y, et al. FOLFIRINOX versus gemcitabine for metastatic pancreatic cancer. N Engl J Med. 2011;364(19):1817-25.

7 Nagrial AM, Chin VT, Sjoquist KM, Pajic M, Horvath LG, Biankin AV, et al. Second-line treatment in inoperable pancreatic adenocarcinoma: a systematic review and synthesis of all clinical trials. Crit Rev Oncol Hematol. 2015;96(3):483-97.

8 Chung VM, McDonough SL, Philip PA, Cardin DB, Wang-Gillam A, Hui L, et al. SWOG S1115: randomized phase II trial of selumetinib (AZD6244; ARRY 142886) hydrogen sulfate (NSC-748727) and MK-2206 (NSC-749607) vs. mFOLFOX in pretreated patients (Pts) with metastatic pancreatic cancer. J Clin Oncol. 2015;33(15_Suppl): 4119.

9 Gill S, Ko YJ, Cripps C, Beaudoin A, Dhesy-Thind S, Zulfiqar M, et al. PANCREOX: a randomized phase III study of fluorouracil/leucovorin with or without oxaliplatin for second-line advanced pancreatic cancer in patients who have received gemcitabine-based chemotherapy. J Clin Oncol. 2016;34(32):3914-20.

10 Chiorean EG, Von Hoff DD, Tabernero J, El-Maraghi R, Ma WW, Reni M, et al. Second-line therapy after nabpaclitaxel plus gemcitabine or after gemcitabine for patients with metastatic pancreatic cancer. $\mathrm{Br} \mathrm{J}$ Cancer. 2016;115(2):188-94.

11 Hubner RA, Cubillo A, Blanc JF, Melisi D, Von Hoff DD, Wang-Gillam A, et al. Quality of life in metastatic pancreatic cancer patients receiving liposomal irinotecan plus 5-fluorouracil and leucovorin. Eur J Cancer. 2019;106:24-33. 